Original Research Paper

\title{
The Roles of Some Antecedents of Broadband User Behavioural Intention among Students in the Rural Areas through PLS-SEM
}

\author{
Ishola D. Muraina, Wan Rozaini Bt Sheik Osman and Azizah Ahmad \\ ITU-UUM ASP CoE for Rural ICT Development/School of Computing, \\ College of Arts and Sciences, Universiti Utara Malaysia, Sintok Kedah, Malaysia
}

\author{
Article history \\ Received: 16-12-2014 \\ Revised: 25-04-2015 \\ Accepted: 07-10-2015 \\ Corresponding Author: \\ Ishola D. Muraina \\ ITU-UUM ASP CoE for Rural \\ ICT Development/School of \\ Computing, College of Arts and \\ Sciences, Universiti Utara \\ Malaysia, Sintok Kedah, \\ Malaysia \\ Email: ishod@uum.edu.my
}

\begin{abstract}
The insufficient broadband enabled-services in the rural areas have been identified among the reasons that prolong the behaviour of rural dwellers on the use of low-band internet while government agencies have started initiations to increase the use of broadband in the rural area. However, previous studies have failed to identify the roles played by antecedents of broadband user behavioural intention in the rural communities. Thus, this research investigates roles of some antecedents of broadband user behavioural intention in the rural areas by adapting UTAUT model. A quantitative research approach was employed by engaging 1,730 participants from 40 villages who are secondary school students that are living in rural areas of northern region of Malaysia. The result reveals that performance expectancy, effort expectancy and compatibility have large effect on broadband user behavioural intention in the rural areas, while social influence recorded medium effect. This implies that all the identified factors may help in determining the behavioural intention of users of broadband in the rural areas.
\end{abstract}

Keywords: Community Informatics, Remote Areas, Rural Dwellers, Determinant Factors, Partial Least Square

\section{Introduction}

The broadband technologies can be grouped into two different types which are wireline broadband and wireless broadband technologies. Wireline broadband technology is comprised of Digital Subscriber Line (DSL), cable modem, power line and fibre optics. Invariably, wireless broadband technology is regarded as Worldwide Interoperability for Microwave Access (WiMax), satellite and Wireless Fidelity (Wi-Fi) (Muraina et al., 2013b). The research has shown that there are variation in the definitions of broadband which is as a result of belief and philosophy of individual and organization. Thus, broadband is considered as the technology that provides consumers fast and always-on access to new applications, services and content with real lifestyle and productivity benefits (Sawyer et al., 2003; Muraina et al., 2013a). Besides, the International Telecommunication Union (ITU) recommendation 1.113 of standardization sector presented broadband as the transmission capacity that is faster than primary rate Integrated Services Digital
Network (ISDN) at 1.5 or 2.0 megabits per second (ICTlogy, 2007; ITU, 2011). Indeed, the society that adopts, adapts and absorbs the benefits of broadbandenabled Information and Communication Technology (ICT), services and applications would be vast in achieving significant benefits in terms of productivity, innovation, growth and quality of life as well as significant competitive advantage over society that do not (BSG Briefing Paper, 2004). This is a result of the innovation brought to the way things are viewed and published by the ICT through the high speed internet (Penalvo et al., 2010).

Many of broadband applications require the minimum speed rate of $50 \mathrm{Mbit} / \mathrm{s}$ in their functionality which is most commonly found in urban areas. On the other hands, application of broadband at the rural or remote areas are still at the struggling stage by depending on the lower speed, causing by high cost of provision, intermittent services and lack of accessibility (Yoshimoto, 2005; Vetter, 2006; Wathen and Roma, 2007). Certainly, the internet users in the rural areas are less in embracing broadband access due to the 
technology, economic and human factors leading them in frequently using low-speed dial up access in lieu of demanding for higher services. This shows that behavioural intention of users of broadband embedded ICT in the rural areas has been affected due to the adaptation of rural dwellers to the low-speed dial up internet (Elizabeth and Alessandra, 2013). Researchers have agitated that there are needs to understand the roles of likely factors that could ignite the behavioural intention of users of broadband among the rural dwellers before selecting the useful factors which is the focus of this research. In order word, behavioural intention is seen as a measure of the strength of one's intention to perform a specified task and find its source from the theory of reasoned action (Ajzen, 1991). Hence, the behavioural intention towards the use of technology comes into existence as a result of roles of some antecedents or factors (Im et al., 2011; Abdulwahab and Zulkhahiri, 2011).

In addition, this research adapts Unified Theory of Acceptance and Use of Technology (UTAUT) model to investigate the roles of some antecedents of behavioural intention of broadband in the rural areas due to its higher variance explanation while compare with other models in the Information Systems (IS) research (Venkatesh et al., 2003; 2011). The UTAUT model is based on the eight models in the domain of IS, while the embedded models are integrated due to their conceptual and empirical factors and focus on the unification of users towards acceptance and usage of technology (Venkatesh et al., 2003). The UTAUT model includes the Technology Acceptance Model (TAM), Theory of Reasoned Action (TRA), Theory of Planned Behavior (TPB), Motivational Model (MM), Combined TAM and TPB (C-TAM-TPB), Innovation Diffusion Theory (IDT), Model of PC Utilization (MPCU) and Social Cognitive Theory (SCT).

\section{Literature Review and Conceptual}

\section{Foundation}

\section{State of Rural Broadband}

There is a widespread belief that information is vital for rural development by using information as a key issue in the information age. Thus, the real challenge of rural areas nowadays is not producing information or storing information, but getting people's behavioural intention towards the information usage (Muraina et al., 2013b). The slow level of development in the rural areas can be improved by the use of ICT to produce information and knowledge society (Juyeon et al., 2012). Moreover, ICT is best fit for bringing about competitive and successful rural community in the effort to better the life of rural dwellers and compete with people in urban areas. Indeed, ICT has been accepted globally for bridging the gaps between urban and rural communities, rich and poor people especially in the area of information seeking. Hence, it is always stress that ICT is useful for the rural communities in interacting with government electronically (e-government) and public sectors with the required infrastructures.

Obviously, the rural ICT solutions are normally offered through internet portals hosted on a delivery web server to provide access for the rural dwellers through inexpensive internet medium with quality and sometimes dial-up internet services. This allows flow of information between the delivery server and the other departments through Intranet/LAN connectivity with servers of those departments which are optional. Due to noncomputerization of back-end systems, the transactions are manually exchanged and response data is keyed in manually through the nodes on the delivery server. It may be noticed that the end-to-end connectivity between the central service providers (district administrations, cooperative unions, post office) and the rural dwellers is accomplished through a number of stages that involves several agencies (Juyeon et al., 2012; Muraina et al., 2012). Therefore, the conditions of ICT in most of rural areas especially the developing countries discourage the users (rural dwellers) leading to the weakening of their behavioural intention towards broadband usage.

Researchers have emphasised that rural dwellers are completely or have less access to the usage of broadband while compare to the people in urban centres which has caused great effect on their education and income (Wan Rozaini et al., 2010; Timothy et al., 2010; Poll, 2008). In a related development, many studies have shown that the current backwardness in the usage of broadband in the rural areas could be traced to the lack of having computer at home which is the antecedents of broadband viability among the rural dwellers (Timothy et al., 2010; Wilson et al., 2003; Bell et al., 2004). The study of Rohaya et al. (2013) emphasised that about 52\% of Sarawak population which is located in the island of Borneo in Malaysia is still living in the rural areas and confronting with great ICT challenges as a result of geographical location or topography. In the context of Africa, the rural dwellers are confronted with lack of internet infrastructure in their communities than the urban dwellers (Francis, 2012). This is as a result of ICT digital divide between the two areas, with $11.6 \%$ internet services at the urban centres and $1.5 \%$ internet services in the rural areas (Francis, 2012). The research by Malaysia Communications and Multimedia Commission (2013) has shown large differences in the internet usage between rural and urban areas in Malaysia as shown in Fig. 1. Comparing the variation between the internet usage among the two domains, the usage of internet in the rural area is lower than that of urban area. 


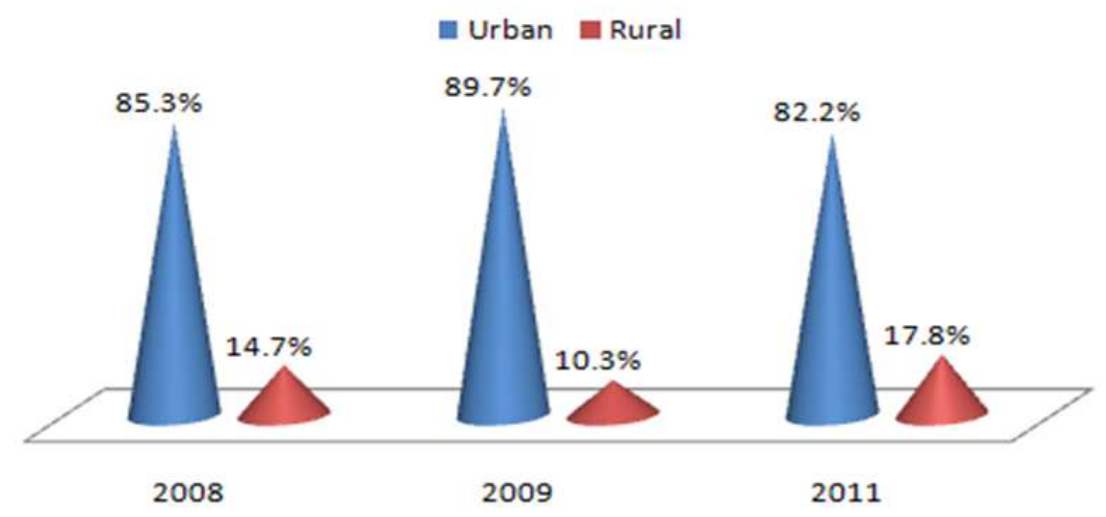

Fig. 1. \% Internet Usage in Urban and Rural Areas in Malaysia in 2008, 2009 and 2011 (Source: MCMC, 2013: Communications and Multimedia Pocket Book of Statistics)

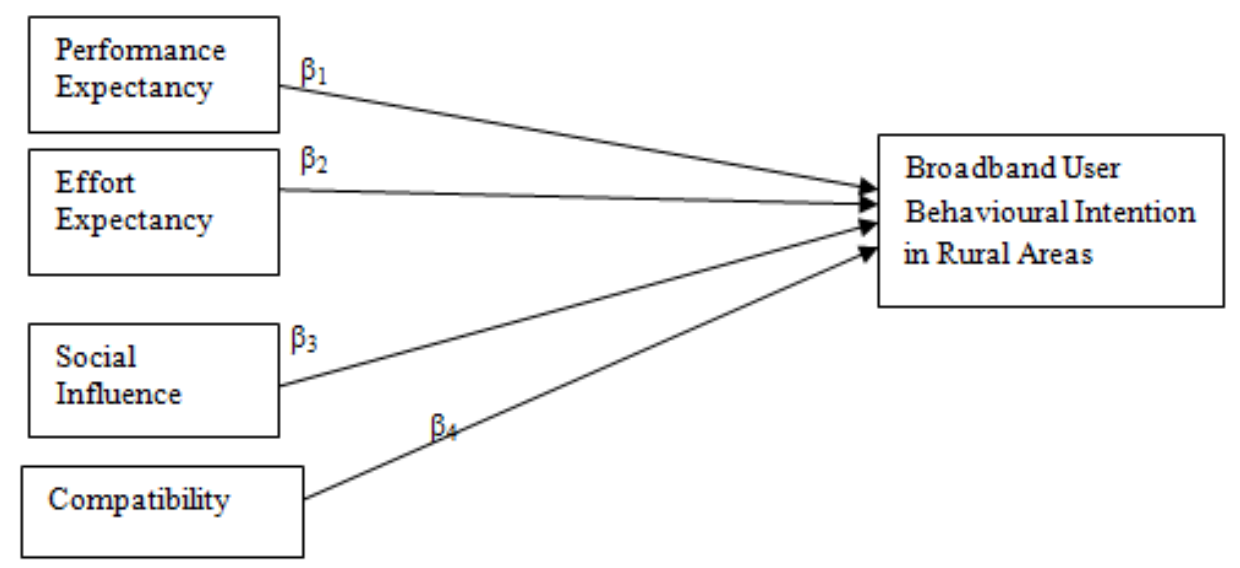

Fig. 2. Research model

\section{Proposed Research Model and Hypotheses Formulation}

The proposed research model in this research has its bases from the UTAUT model as a result of its ability to explain variation in the acceptance and usage of technology. Thus, this research adapts some of its constructs while compatibility is added based on the stands of some researchers in order to investigate the roles of antecedents of broadband user behavioural intention in the rural areas as shown in Fig. 2.

The performance expectancy is described as the degree to which an individual believes that using the system or device assist to achieve the job performance (Venkatesh et al., 2003). Many researchers have agreed to the outcome derived from some studies conducted using UTAUT model and concluded that effort expectancy was found to be positively related to behavioural intention towards usage of ICT devices (Park et al., 2007; Im et al., 2011). Moreover, effort expectancy as one of the exogenous constructs in
UTAUT model can be described as the degree of ease of use of information system or devices felt by a user. Besides, Muraina et al. (2012), Taylor and Todd (1995) emphasised that effort expectancy influences the behavioural intention towards acceptance and usage of information technology devices more than one time. In addition, the degree to which an individual user feels that other people believe that he or she should be using the ICT devices is regarded as social influence (Venkatesh et al., 2003). Thus, social influence posted a significantly influence on the behavioural intention towards the ICT from time to time (Suha and Anne, 2008).

Compatibility is the extent by which innovation is envisage as being consistent with the values, needs and experiences of future adopters or users (Moore and Benbasat, 1991). The opinion of Rogers (2010) towards compatibility is the degree to which an innovation is perceived to be consistent with the current values, past experiences and needs of the future adopters. In other words, Rogers (2003) established that compatibility is the degree by which individual that involved in online 
transactions through the use of mobile commerce feels consistent with the future users of the existing values, beliefs, initial knowledge and current needs. Indeed, compatibility is considered as one of the important determinant's factors of the behavioural intention towards of use of devices or technologies ( $\mathrm{Wu}$ and Wang, 2005). Hence, the following hypotheses are formulated based on the previous studies in order to investigate the likely roles of performance expectancy, effort expectancy, social influence and compatibility as antecedents of broadband user behavioural intention in the rural areas:

$\mathrm{H}_{1}$ : Performance expectancy relates to the broadband user behavioural intention in the rural areas.

$\mathrm{H}_{2}$ : Effort expectancy relates to the broadband user behavioural intention in the rural areas.

$\mathrm{H}_{3}$ : Social influence relates to the broadband user behavioural intention in the rural areas.

$\mathrm{H}_{4}$ : Compatibility relates to the broadband user behavioural intention in the rural areas.

\section{Materials and Methods}

This research uses Partial Least Square (PLS) Structural Equation Model (SEM) to validate the antecedents of broadband user behavioural intention in the rural areas due to its efficiency to simplify the multivariate complex model as well as handling non normal distributed data (Michael and Andreas, 2004; Hair et al., 2011). Besides, Hair et al. (2011) stress that PLS-SEM is worth of using in the multivariate model because it bounds to bring additional value to the explained variance of dependent variable (Broadband Behavioural Intention).

\section{Measurement}

All the four constructs that were used in this research as antecedent factors of broadband user behavioural intention in the rural areas were measured by using 5 point likert scale survey questionnaire, ranging from strongly disagree (1) to strongly agree (5). We built on UTAUT measurement items while some items that used to measure performance expectancy and effort expectancy were adapted from (Venkatesh et al., 2003; 2011; 2012; Abdulwahab and Zulkhairi, 2011). Moreover, social influence was measured by adapting items from (Venkatesh et al., 2003; Abdulwahab and Zulkhairi, 2012). Lastly, compatibility construct was measured by adapting items from Moore and Benbasat (1991) and Rogers (2003).

\section{Location, Participants and Data Collection Technique}

This research was conducted in the rural areas of northern region of Malaysia due to their slow rate of development when compared with the rest of regions in Malaysia. Each of the covered rural areas has their population below 10,000 inhabitants per kilometre with some agricultural features, forest and water bodies. Besides that, 1,820 participants who are secondary school students from 40 villages were invited to the rural internet centres that were used as the distribution and collation of questionnaires and are located at the strategic locations in the northern region of Malaysia. Indeed, only 1,730 participants were eventually selected for administering our survey questionnaires through fish-bowl style of simple random sampling. The data collection took exactly 9 weeks as there were early and late respondents. Prior to the selection of the 1,730 participants in the data collection process, we explained the meaning of broadband to the participants and found that they are users of broadband either at their school, home, cybercafé or rural internet centres. Moreover, 1,689 questionnaires were returned to the rural internet centres after completion by the participants, representing response rate of $97.63 \%$. Following this further, we checked for non response bias after collection of the data and found no differences in demographics between respondents and non-respondents.

\section{Results}

\section{Data Preparation}

Prior to the data analysis using PLS-SEM for the collected data, we ensured that data was properly screened for the missing values by using SPSS version 20 . The analysis performed routine check on the outliers which can distort or turn the outcome of the research to unacceptable issue. Our data passed both error and interesting outliers while 36 cases failed to certify influential outliers, leading to discarding of 36 cases from 1,689 returned questionnaires. Thus, the remaining 1,653 screened questionnaires found fit for use in the subsequent analysis. Influential outliers' cases are recommended to remove from the entire cases for the multivariate analysis due to its ability to affect model fitness (Aguinis et al., 2013). Moreover, the test of normality was performed and found that dataset was not normal, calling for the use of PLS-SEM as suggested by Hair et al. (2011). The test of multicollinearity was conducted by observing values of Tolerance and Variance Inflation Factor (VIF). The results showed that all the Tolerance values are above 0.10 and VIF values are less than 10, showing that our data fulfils multicollinearity conditions.

\section{Background of the Respondents}

The total number of $52.6 \%$ of the respondents was male students while $47.4 \%$ were female students with 
average age of 16 years. The data gathered shows that $68.4 \%$ of the respondents are in grades $10-11,18.4 \%$ in grades $7-9$ and $13.2 \%$ in grade 12 of the secondary school education. Moreover, $50 \%$ of the respondents confirmed that they are leaving with their parents during the academic session, while others are staying at the boarding house and with the legal guardians. Indeed, $57.9 \%$ of the respondents claimed that their parents' educational levels is secondary school education, $13.2 \%$ bachelor degree, $2.6 \%$ master degree, while $26.3 \%$ of the respondents' parents do not have educational background. Besides, $18.4 \%$ of the respondents' parents engage in rubber tapping activity as source of income, $21.1 \%$ engage in small scale businesses, $5.3 \%$ work in industry and $55.3 \%$ engage in other activities.

In addition, majority of $52.6 \%$ of the respondents say they do not have computer at home, while $65.8 \%$ do not have internet connectivity at home due to the high starting cost, lack of equipment and limited coverage of internet in their villages. Meanwhile, all the respondents were found familiar with the usage of broadband either at home, school, rural internet centres or cybercafé. However, $47.4 \%$ are still using dial-up internet while $52.6 \%$ are using broadband internet. This is an indication of needs to search for the factors that could influential broadband user behavioural intention in the rural areas.

\section{Partial Least Square Approach}

Partial Least Square (PLS) is used to investigate the roles of antecedent of broadband user behavioural intention in the rural areas as a result of many interactions between the constructs in the research model, shown in Fig. 2. The use of PLS-SEM for the research objective examined measurement model in order to assess reliability and validity of the constructs as recommended by (Chin et al., 2003). Consequently, the structural model was examined to assess relationship between the exogenous and endogenous variables and confirmed the set hypotheses.

\section{Measurement Model}

The results of measurement model are shown in Table 1-4 representing the reliability and validation of the constructs that used to determine the broadband user behavioural intention in the rural areas.
From the Table 1, the internal consistency of all the constructs in the model shown in Fig. 2 ranges from 0.7265 to 0.7946 which is acceptable and revealed that the scales used in the instrument were reliable. The Average Variance Extracted (AVE) is greater than 0.50 threshold value, meaning that the constructs passed the convergent validity based on Fornell and Larcker (1981).

Moreover, it shows in Table 2 that the indicator loading of all the items are above 0.70 benchmark value which indicate that the loading items supported internal consistency.

Besides that, the square root of AVE of each construct in the model is higher than the corresponding constructs in the column of discriminant validity using Fornell-Larcker criterion as shown in Table 3.

Indeed, the discriminant validity through the crossloading approach was conducted on the collected data and revealed in Table 4 that all the values of items in each construct are higher than their corresponding items. Hence, all the rules of measurement model are achieved thus fit to proceed for structural model assessment.

\section{Structural Model}

The structural model was assessed by using the bootstrapping method. Hair et al. (2011) described bootstrapping method as the use of repeated approach to obtain replacement from the main sample towards achieving the standard error for hypothesis testing. Thus, our research uses bootstrapping method through the Smart-PLS 2.0 with 5,000 iterations for 1,653 cases. The obtained results show that all the path coefficients in our model are significant and supported the set hypotheses, shown in Table 5. Indeed, our model on the antecedents of broadband user behavioural intention is fit and relevant for the rural areas with the variance explanation $\left(R^{2}\right) 0.559$ as shown in Table 1.

Pursuing this further, it becomes an important step to investigate the role played by each of the exogenous variables which are antecedents of broadband user behavioural intention in the rural areas in our research. This is achieved by examined the effect size $\left(f^{2}\right)$ which estimates the impact of the exogenous variables on the endogenous variable. Researchers have continuously emphasised that the effect size of exogenous variable expresses the impacts or roles played by each exogenous construct on the endogenous variable (Hair et al., 2013; 2011; Chin, 2010).

Table 1. Average variance extracted and composite reliability

\begin{tabular}{llllll}
\hline Variables & AVE & C R & $\mathrm{R}^{2}$ & C A & Communality \\
\hline Broadband user Behavioural intention & 0.5422 & 0.8209 & 0.7585 & 0.7265 & 0.5422 \\
Compatibility & 0.5821 & 0.8476 & 0.0000 & 0.7696 & 0.5821 \\
Effort expectancy & 0.5553 & 0.8510 & 0.0000 & 0.7946 & 0.5553 \\
Performance expectancy & 0.5636 & 0.8329 & 0.0000 & 0.7310 & 0.5636 \\
Social influence & 0.7393 & 0.8500 & 0.0000 & 0.7503 & 0.7393 \\
\hline
\end{tabular}


Ishola D. Muraina et al. / American Journal of Applied Sciences 2015, 12 (11): 820.829 DOI: $10.3844 /$ ajassp.2015.820.829

Table 2. Outer loading

\begin{tabular}{|c|c|c|c|c|c|}
\hline & BUBI & $\mathrm{CP}$ & $\mathrm{EE}$ & $\mathrm{PE}$ & SI \\
\hline BUBI1 & 0.7832 & & & & \\
\hline BUBI2 & 0.7292 & & & & \\
\hline BUBI3 & 0.9249 & & & & \\
\hline BUBI4 & 0.7598 & & & & \\
\hline $\mathrm{CP} 1$ & & 0.7668 & & & \\
\hline $\mathrm{CP} 2$ & & 0.7405 & & & \\
\hline CP3 & & 0.7316 & & & \\
\hline $\mathrm{CP} 4$ & & 0.8105 & & & \\
\hline EE1 & & & 0.7988 & & \\
\hline EE2 & & & 0.8643 & & \\
\hline EE3 & & & 0.7955 & & \\
\hline EE4 & & & 0.8204 & & \\
\hline EE5 & & & 0.8851 & & \\
\hline PE1 & & & & 0.7467 & \\
\hline PE2 & & & & 0.8879 & \\
\hline PE3 & & & & 0.8565 & \\
\hline PE5 & & & & 0.8585 & \\
\hline SI2 & & & & & 0.8304 \\
\hline SI4 & & & & & 0.8883 \\
\hline Variables & BUBI & $\mathrm{CP}$ & EE & PE & SI \\
\hline$\overline{\text { BUBI }}$ & 0.7363 & & & & \\
\hline $\mathrm{CP}$ & 0.6984 & 0.7630 & & & \\
\hline $\mathrm{EE}$ & 0.5917 & 0.5582 & 0.7452 & & \\
\hline PE & 0.4831 & 0.5207 & 0.6913 & 0.7507 & \\
\hline SI & 0.3817 & 0.4528 & 0.1516 & 0.2183 & 0.8598 \\
\hline
\end{tabular}

Table 4. Discriminant validity (cross loading)

\begin{tabular}{llllrr}
\hline & BUBI & CP & EE & PE & SI \\
\hline BUBI1 & 0.5832 & 0.4829 & -0.0267 & -0.0664 & 0.3581 \\
BUBI2 & 0.6292 & 0.3778 & 0.1544 & 0.0759 & 0.0780 \\
BUBI3 & 0.9249 & 0.6995 & 0.7065 & 0.5787 & 0.2579 \\
BUBI4 & 0.7598 & 0.4481 & 0.5784 & 0.5231 & 0.1625 \\
CP1 & 0.4781 & 0.7668 & 0.3850 & 0.2414 & 0.3753 \\
CP2 & 0.4577 & 0.7405 & 0.1872 & 0.3826 & 0.2022 \\
CP3 & 0.3674 & 0.7316 & 0.3810 & 0.6715 & 0.2013 \\
CP4 & 0.7160 & 0.8105 & 0.6397 & 0.0387 & 0.5001 \\
EE1 & 0.0049 & 0.2661 & 0.2988 & 0.4978 & 0.2105 \\
EE2 & 0.5114 & 0.3757 & 0.8643 & 0.5975 & 0.1127 \\
EE3 & 0.3330 & 0.3717 & 0.6955 & 0.4632 & 0.1066 \\
EE4 & 0.4436 & 0.3932 & 0.8204 & 0.7105 & 0.0301 \\
EE5 & 0.5997 & 0.6413 & 0.8851 & 0.5467 & 0.2210 \\
PE1 & 0.2282 & 0.4723 & 0.2888 & 0.8879 & 0.0148 \\
PE2 & 0.4786 & 0.5532 & 0.6503 & 0.8565 & 0.3227 \\
PE3 & 0.4168 & 0.3161 & 0.6111 & 0.6585 & 0.1087 \\
PE5 & 0.2512 & 0.2148 & 0.4353 & 0.2121 & 0.1232 \\
SI2 & 0.2947 & 0.3626 & 0.1796 & 0.1686 & 0.8304 \\
SI4 & 0.3574 & 0.4133 & 0.0904 & & 0.8883 \\
\hline
\end{tabular}

Table 5. Significance of the path coefficients

\begin{tabular}{|c|c|c|c|c|c|}
\hline Hypotheses & Path & Relationships & Original sample & T-Statistics & Remarks \\
\hline $\mathrm{H}_{1}$ & $\beta_{1}$ & PE -> BUBI & 0.3216 & $2.323^{*}$ & Supported \\
\hline $\mathrm{H}_{2}$ & $\beta_{2}$ & EE -> BUBI & 0.3149 & $2.133^{*}$ & Supported \\
\hline $\mathrm{H}_{3}$ & $\beta_{3}$ & SI -> BUBI & 0.4223 & $2.6526^{*}$ & Supported \\
\hline $\mathrm{H}_{4}$ & $\beta_{4}$ & CP -> BUBI & 0.4705 & $2.841^{*}$ & Supported \\
\hline
\end{tabular}

Degree of freedom: 5,$000 ; *$ significant at $\mathrm{p}<0.05$ (2-tailed test) 
Table 6. Effect size of the model

\begin{tabular}{llll}
\hline Correlation between dimension & Path coefficients & Effect size $\left(\mathrm{f}^{2}\right)$ & Remarks \\
\hline PE $->$ BUBI & 0.3216 & 0.352 & Large \\
EE $->$ BUBI & 0.3149 & 0.415 & Large \\
SI -> BUBI & 0.4223 & 0.295 & Medium \\
CP -> BUBI & 0.4705 & 0.364 & Large \\
\hline
\end{tabular}

Precisely, the $f^{2}$ for the research model was assessed by removing and replacing a particular exogenous variable so as to investigate the roles play by the exogenous variables towards broadband user behavioural intention in the rural areas and mathematically shown in Equation 1:

$$
f^{2}=\frac{R_{\text {included }}^{2}-R_{\text {excluded }}^{2}}{1-R_{\text {included }}^{2}}
$$

Where:

$$
\begin{aligned}
f^{2}= & \text { The effect size of } R^{2} \text { when a particular } \\
& \text { exogenous variable is present and omitted in } \\
& \text { the model } \\
R_{\text {included }}^{2}= & \text { The value of } R^{2} \text { when all the exogenous } \\
& \text { variables are existed } \\
R_{\text {excluded }}^{2}= & \text { The value of } R^{2} \text { when a particular exogenous } \\
& \text { variable is omitted from the model }
\end{aligned}
$$

Moreover, the effect sizes of PE -> BUBI, EE -> BUBI, SI -> UBI and CP -> BUBI are shown in Table 6. Meanwhile, the effect sizes are categorised based on the recommended benchmark values of Chin (1998) as 0.02 (small), 0.15 (medium) and 0.35 (large). The results in Table 6 reveals that effect of PE -> BUBI, EE -> BUBI and $\mathrm{CP}->$ BUBI are large, while SI $->$ BUBI is medium.

\section{Discussion}

This research adapts the use of UTAUT model in investigating the roles of some antecedents towards the broadband user behavioural intention in the rural areas. Based on the review of previous studies, the research finally resulted in identifying Performance Expectancy (PE), Effort Expectancy (EE), Social Influence (SI) and Compatibility (CP) as the antecedents of broadband user behavioural intention in the rural areas. The results in Table 5 showed that there is relationship between Performance Expectancy (PE) and the Broadband User Behavioural Intention (BUBI) in the rural areas. The result portrays the opinion of rural dwellers in northern region of Malaysia as they accept that believe of individual can affect the broadband user behavioural intention in their communities. The situation is not different in Orlu zone which comprises of many rural areas in Imo state part of Nigeria as they supported that belief of expected users of ICT devices can contribute to the decision making towards usage and excel in their activities (Alphonsus et al., 2011). Looking at the implication of this theme in the western part of the world, Adria and Brown (2012) concluded in their finding that belief of rural dwellers in Canada on the use of broadband has effect on the behavioural intention. Indeed, this research has shown that $\mathrm{PE}$ plays an important role on the BUBI in the rural areas with large effect size. This reveals that the rural dwellers would be more productive in their job activities with strong PE towards BUBI.

Furthermore, the Effort Expectancy (EE) which is the degree of ease attached to the usage of broadband. Therefore, the research shows that there is significant relationship between effort expectancy and broadband user behavioural intention in the rural areas which supports the set hypothesis $\left(\mathrm{H}_{2}\right)$. The implication of this is that the more the ease of use of broadband among the rural dwellers the more their positive intention towards the use of broadband. The fulfillment of hypothesis $\left(\mathrm{H}_{2}\right)$ becomes reality as a result of educational background of the participants in this research. This is in line with the study of Furuholt and Kristiansen (2007) who reveals that irrespective of marginalisation of rural dwellers in Tanzania (a country in Sub-Sahara Africa); many of them still intend to use the fast internet due to its simplified nature. A related research by Adria and Brown (2012) stressed that the rural broadband becomes a necessity for the people of Alberta rural community in Canada based on their behavioural intention built towards it as they found it easy to use. Hence, this research reveals that effort expectancy is one of the factors that could play a large role in achieving broadband behavioural intention in the rural areas.

In addition, the research found that the relationship between Social Influence (SI) and Broadband User Behavioural Intention (BUBI) is significant and supported the hypothesis $\left(\mathrm{H}_{3}\right)$ but with medium effect. This explains that the sustainability of positive broadband user behavioural intention in the rural areas could be triggered by the feelings of users that some important people perceived he or she should use broadband in their communities. Moreover, Compatibility (CP) is related to Broadband User Behavioural Intention (BUBI) in the rural areas as reveals by this research. The outcome of the research shows that if the rural broadband is compatible with the activities the rural dwellers accepts it for, their 
behavioural intention would be constructive. Indeed, researchers have emphasised that relevancy or compatibility of technology is enough to serve as factor for the users at rural Bangladesh to decide on its usage (Sirajul and Suha, 2008). Besides, the effect of relationship between compatibility $(\mathrm{CP})$ and broadband user behavioural intention is large as shown in Table 6 .

\section{Conclusion}

This research investigates the roles played by some antecedents towards the broadband user behavioural intention in the rural areas by adapting UTAUT model. The results were obtained by using the Smart PLS 2.0 and SPSS version 20 leading to the fact that the identified antecedents; performance expectancy, effort expectancy, social influence and compatibility were significant as antecedents of broadband user behavioural intention in the rural areas. Besides, the research showed that performance expectancy, effort expectancy and compatibility had large effect size towards the exogenous variable broadband user behavioural intention, while social influence possess medium effect. Moreover, our research model as shown in Fig. 2 is capable of using globally while investigating or determining the broadband or ICT user behavioural intention in the rural areas. This is because of the applications of our results to the contemporary studies in ICT or broadband in both developing and developed countries as explained in the discussion section. Hence, this research would be further studied in future on the implication of broadband user behavioural intention on the actual use of broadband in the rural areas.

\section{Acknowledgement}

The ultimate appreciation is due to ITU-UUM ASP CoE for Rural ICT Development for their support and grants provided for this research. Besides, we thank the corresponding author and co-authors for their immensely contribution towards the success of this research.

\section{Funding Information}

Authors like to thank ITU-UUM ASP CoE for Rural ICT Development for funding this research work.

\section{Author's Contributions}

Ishola D. Muraina: Contributed to this manuscript through the planning and conduction of the research. He also coordinated the data collection, analysis and manuscript writing.

Wan Rozaini Sheik Osman: Participated in the planning of the research, participation in data collection and manuscript writing.
Azizah Ahmad: Engaged in the planning of the research, participation in the data collection and manuscript writing.

\section{Ethics}

This is the original research work and contains unpublished material. Therefore, the corresponding author confirms that co-authors have read and approved the manuscript with no ethical issues involved.

\section{References}

Abdulwahab, L. and M.D. Zulkhairi, 2011. Effectiveness of telecentre using a model of Unified Theory of Acceptance and Use of Technology (UTAUT): Structural equation modeling approach. J. Emerg. Trend Comput. Inform. Sci., 2: 402-410.

Abdulwahab, L. and M.D. Zulkhairi, 2012. Modeling the determinants and gender, age and ethnicity difference in telecommunication centre acceptance. Res. J. Inform. Technol., 4: 85-105. DOI: $10.3923 /$ rjit.2012.85.105

Adria, M. and D. Brown, 2012. Ambiguity and uncertainty in the "last mile": Using sense-making to explore how rural broadband networks are created. J. Community Inform., 8: 1-15.

Aguinis, H., R.K. Gottfredson and H. Joo, 2013. Bestpractice recommendations for defining, identifying and handling outliers. Organ. Res. Methods, 16: 270-301. DOI: $10.1177 / 1094428112470848$

Ajzen, I., 1991. The theory of planned behavior. Organ. Behav. Hum. Decision Process., 50: 179-211. DOI: 10.1016/0749-5978(91)90020-T

Alphonsus, O.A., A.U. Elochukwu and I. Sylvanus, 2011. Internet penetration and diffusion in orlu zone of Imo state, Nigeria: Prospects and bottlenecks. Asian J. Inform. Technol., 10: 149-153. DOI: 10.3923/ajit.2011.149.153

Bell, P., P. Reddy and L. Rainie, 2004. Rural Areas and The Internet: Rural Americans' Internet Use Has Grown, But They Continue to Lag Behind Others. 1st Edn., Pew Internet and American Life Project, pp: 38.

BSG Briefing Paper, 2004. The impact of broadbandenabled ICT, content, applications and services on the UK economy and society to 2010 .

Chin, W.W., 1998. The Partial Least Squares Approach to Structural Equation Modeling. In: Modern Methods for Business Research, Marcoulides, G.A. (Ed.), Psychology Press, ISBN-10: 1135684138, pp: 295-358.

Chin, W.W., 2010. How to Write Up and Report PLS Analysis? In: Handbook of Partial Least Squares: Concepts, Methods and Applications, Vinzi, V.E., W.W. Chin, J. Henseler and H. Wang (Eds.), Springer Science and Business Media, Berlin, ISBN-10: 3540328270, pp: 655-690. 
Chin, W.W., B.L. Marcolin and P.N. Newsted, 2003. A partial least squares latent variable modeling approach for measuring interaction effects: Results from a Monte Carlo simulation study and an electronic-mail emotion/adoption study. Inform. Syst. Res., 14: 189-217.

DOI: $10.1287 /$ isre. 14.2.189.16018

Elizabeth, M. and F. Alessandra, 2013. Productivity and broadband: The human factor. Int. Regional Sci. Rev., 36: 392-423. DOI: $10.1177 / 0160017612471191$

Fornell, C. and D.F. Larcker, 1981. Structural equation models with unobservable variables and measurement error: Algebra and statistics. J. Market. Res., 18: 328-388. DOI: 10.2307/3150980

Francis, O., 2012. Internet access in Nigeria: Perception of National Open University of Nigerian (NOUN) students. Int. J. Emerg. Technol. Adv. Eng., 2: 492-497.

Furuholt, B. and S. Kristiansen, 2007. A rural-urban digital divide? Regional aspects of internet use in Tanzania. Proceedings of the International Conference on Social Implications of Computers in Developing Countries, (CDC' 07), pp: 1-14.

Hair, J.F., G.T.M. Hult, C. Ringle and M. Sarstedt, 2013. A Primer on Partial Least Squares Structural Equation Modeling (PLS-SEM). SAGE Publications, Los Angeles, ISBN-10: 1452217440, pp: 328.

Hair, J.F., C.M. Ringle and M. Sarstedt, 2011. PLSSEM: Indeed a silver bullet. J. Market. Theory Practice, 19: 139-151.

Poll, H., 2008. Number of "cyberchondriacs"-Adults who have Ever Gone Online for Health InformationHas Plateaued Or Declined. 1st Edn., Harris Interactive, Rochester, NY, pp: 6.

ICTlogy, 2007. Retrieved.

Im, I.I., S. Hong and M. Kang, 2011. An international comparison of technology adoption: Testing the UTAUT model. Inform. Manage., 48: 1-8. DOI: $10.1016 /$ j.im.2010.09.001

ITU, 2011. Birth of Broadband.

Juyeon, H., P. Jihun, L.J. Nam and Y.M. Jae, 2012. Understanding continuous use of virtual communities: A comparison of four theoretical perspectives. Proceedings of 45th Hawaii International Conference on System Sciences, (CSS' 12), pp: 753-762.

Michael, H. and M.K. Andreas, 2004. A beginner's guide to partial least squares analysis. Understand. Statist., 3: 283-297. DOI: $10.1207 / \mathrm{s} 1532803$ lus0304 4

Moore, G.C. and I. Benbasat, 1991. Development of an instrument to measure the perceptions of adopting an information technology innovation. Inform. Syst. Res., 2: 192-222. DOI: 10.1287/isre.2.3.192
Muraina, I.D., S.A.W. Rozaini and A. Azizah, 2012. Conceptualising service quality and technology factor as drivers for continuous usage of broadband among rural dwellers in Malaysia. Proceedings of 3rd International Conference on Network Applications, Protocols and Services, Sept. 19-21, Kuala Lumpur, pp: 83-87.

Muraina, I.D., R.S.O. Wan and A. Azizah, 2013a. Efficacy of UTAUT model in continuation of usage of broadband in the rural areas of northern region of Malaysia. Proceedings of International Conference on Rural ICT Development, Jun. 25-27, Malacca, Malaysia, pp: 174-179.

Muraina, I.D., R.S.O. Wan and A. Azizah, 2013b. Investigating the readiness of broadband continuous usage among rural dwellers in the northern region of Malaysia. Int. J. Comput. Commun. Eng., 2: 679-683. DOI: 10.7763/IJCCE.2013.V2.273

Park, J., S. Yang and X. Lehto, 2007. Adoption of mobile technology for Chinese consumers. J. Electr. Commerce Res., 8: 196-205.

Penalvo, F.J.G., C.G. Figuerola and J.A. Merlo, 2010. Open knowledge: Challenges and facts. Online Inform. Rev., 34: 520-539. DOI: $10.1108 / 14684521011072963$

Rogers, E.M., 2010. The Diffusion of Innovations. 4th Edn., Simon and Schuster, New York, ISBN-10: 1451602472, pp: 518.

Rogers, E.M., 2003. Diffusion of Innovations. 5th Edn., Simon and Schuster, New York, ISBN-10: 0743258231, pp: 576.

Rohaya, M.N., E.C. Teddy and R.J.W. Constance, 2013. Malaysian rural community as consumer of health information and their use of ICT. Malaysian J. Commun., 29: 161-178.

Sawyer, S., J.P. Allen and L. Heejin, 2003. Broadband and mobile opportunities: A socio-technical perspective. J. Inform. Technol., 18: 121-136. DOI: $10.1080 / 0268396032000101171$

Sirajul, M.I. and A. Suha, 2008. Sustainable rural IS implementation process for developing countries: Agriculture Market Information Services (AMIS) in Bangladesh. Proceedings of the 1st International Conference on M4D Mobile Communication Technology for Development, Dec. 11-12, Karlstad, Sweden, pp: 127-137.

Suha, A. and M. Anne, 2008. The use of the UTAUT model in the adoption of e-government services in Kuwait. Proceedings of the 41st Annual Hawaii International Conference on System Sciences, Jan. 710, IEEE Xploer Press, Waikoloa, HI, pp: 219-230. DOI: 10.1109/HICSS.2008.452

Taylor, S. and P. Todd, 1995. Understanding information technology usage: A test of competing models. Inform. Syst. Res., 6: 144-176.

DOI: $10.1287 /$ isre.6.2.144 
Timothy, M.H., R.C. Shelia, D. Patricia and G. Melinda, 2010. Rural-urban differences in general and health-related internet use. Am. Behavioural Sci., 53: 1304-1325. DOI: 10.1177/0002764210361685

Venkatesh, V., M. Morris, G. Davis and F. Davis, 2003. User acceptance of information technology: Toward a unified view. Inform. Syst. Q., 27: 425-478.

Venkatesh, V., T. Sykes and X. Zhang, 2011. Just what the doctor ordered: A reviewed UTAUT for EMR system adoption and use by doctor. Proceedings of the 44th Huwaii International Conference on System Sciences, Kauai, HI, USA., pp: 1-10.

DOI: 10.1109/HICSS.2011.1

Venkatesh, V., J.Y.L. Thong and X. Xu, 2012. Consumer acceptance and use of information technology: Extending the unified theory of acceptance and use of technology. MIS Q., 36: 157-178.

Vetter, P., 2006. The role of wireline access technology to bridge the digital divide. MUSE IST Workshop, Brussels, Belgium.
Wan Rozaini, S.O., Y. Yuhanis, M. Zahurin and H. Rohani, 2010. Rural ICT development. Proceeding of 3rd National Conference on Rural Information Communication Technology Development, (CTD' 10), Publication Malaysia.

Wathen, C.N. and M.H. Roma, 2007. "I try to take care of it myself." How rural women search for health information. Qualitative Health Res., 17: 639-651. DOI: $10.1177 / 1049732307301236$

Wilson, K., J. Wallin and C. Reiser, 2003. Social stratification and the digital divide. Social Sci. Comput. Rev., 21: 133-143. DOI: $10.1177 / 0894439303021002001$

$\mathrm{Wu}$, J.H. and S.C. Wang, 2005. What drives mobile commerce?: An empirical evaluation of the revised technology acceptance model. Inform. Manage., 42: 719-729. DOI: 10.1016/j.im.2004.07.001

Yoshimoto, N., 2005. NTT's deployment of FTTH services and future developments. FTTH Conference, USA. 